\title{
Eficacia de soluciones salinas modificadas para la supervivencia de plerocercoides Tetraphyllidea de concha de abanico (Argopecten purpuratus)
}

\author{
Efficacy of a modified saline solutions for the survival of Tetraphyllidean \\ plerocercoids from the scallop (Argopecten purpuratus)
}

\author{
Teresa Castro ${ }^{1,3}$, Enrique C. Mateo ${ }^{1}$, César A. Peña ${ }^{2}$
}

\section{Resumen}

\begin{abstract}
El objetivo de este estudio fue obtener una solución salina modificada que permita una supervivencia más prolongada para el estudio morfológico de plerocercoides Tetrafilideos de la concha de abanico Argopecten purpuratus. Se evaluaron dos temperaturas: T-1, temperatura ambiente y T-2, temperatura constante de $0.5^{\circ} \mathrm{C}$ y cinco soluciones: $\mathrm{A}=\mathrm{SSF}$ (solución salina fisiológica $-\mathrm{SSF}-$ al $0.85 \%$ ), $\mathrm{B}=1 / 4 \mathrm{AM}$ (agua de mar) + 3/4 $\mathrm{SSF}, \mathrm{C}=1 / 2 \mathrm{AM}+1 / 2 \mathrm{SSF}, \mathrm{D}=3 / 4 \mathrm{AM}+1 / 4 \mathrm{SSF}, \mathrm{E}=\mathrm{AM}$. Se utilizaron 100 larvas vivas recién extraídas de gónadas de $A$. purpuratus (10 por grupo experimental) durante 13 días. La mayor supervivencia se observó en las soluciones $\mathrm{B}$ y C del T-1, donde permanecieron vivos cuatro plerocercoides hasta el día 10 y tres hasta el día 12, respectivamente. Con excepción de la solución A, las larvas mantuvieron su actividad y forma normal en el resto de las soluciones hasta el día 6. Este trabajo demuestra que las soluciones salinas usua-
\end{abstract}

${ }^{1}$ Laboratorio de Patobiología Acuática, Dirección General de Investigaciones en Acuicultura, Instituto del Mar del Perú (IMARPE), Callao, Perú

${ }^{2}$ Laboratorio Experimental de Patobiología Acuática, Departamento Académico de Acuicultura, Facultad de Oceanografia, Pesquería, Ciencias Alimentarias y Acuicultura, Universidad Nacional Federico Villarreal, Lima, Perú

${ }^{3}$ E-mail: tcastro@imarpe.gob.pe; https://orcid.org/0000-0002-9094-400X

Recibido: 11 de mayo de 2020

Aceptado para publicación; 28 de noviembre de 2020

Publicado: 23 de junio de 2021

CLos autores. Este artículo es publicado por la Rev Inv Vet Perú de la Facultad de Medicina Veterinaria, Universidad Nacional Mayor de San Marcos. Este es un artículo de acceso abierto, distribuido bajo los términos de la licencia Creative Commons Atribución 4.0 Internacional (CC BY 4.0) [https:// creativecommons.org/licenses/by/4.0/deed.es] que permite el uso, distribución y reproducción en cualquier medio, siempre que la obra original sea debidamente citada de su fuente original 
les $(0.75$ a $0.9 \%)$ pueden no ser óptimas para mantener plerocercoides marinos vivos y que una salinidad que se aproxima al agua de mar es más apropiada.

Palabras clave: solución salina, plerocercoide, supervivencia, concha de abanico, Argopecten purpuratus

\section{Abstract}

The aim of this study was to obtain a modified saline solution that allows a longer survival for the morphological study of Tetraphyllideans plerocercoids from the scallop Argopecten purpuratus. Two temperatures were evaluated: T-1, room temperature and $\mathrm{T}-2$, constant temperature of $0.5{ }^{\circ} \mathrm{C}$ and five solutions: A $=\mathrm{FSS}$ (physiological saline solution $-\mathrm{FSS}-0.85 \%$ ), $\mathrm{B}=1 / 4 \mathrm{SW}$ (sea water) $+3 / 4 \mathrm{FSS}, \mathrm{C}=1 / 2 \mathrm{SW}+1 / 2 \mathrm{FSS}, \mathrm{D}=3 / 4 \mathrm{SW}$ $+1 / 4 \mathrm{FSS}, \mathrm{E}=\mathrm{SW}$. One hundred live larvae freshly extracted from $A$. purpuratus gonads (10 per experimental group) were used for 13 days. The longest survival was observed in $\mathrm{T}-1$ solutions $\mathrm{B}$ and $\mathrm{C}$, where four plerocercoids remained alive until day 10 and three until day 12 , respectively. With the exception of solution $A$, the larvae maintained their normal activity and shape in the rest of the solutions until day 6 . This work shows that the usual saline solutions $(0.75$ to $0.9 \%)$ may not be optimal for keeping marine plerocercoids alive and that a salinity approaching seawater is more appropriate.

Key words: saline solution, plerocercoid, survival, scallop, Argopecten purpuratus

\section{INTRODUCCIÓN}

Se cree que el $\mathrm{NaCl}$ al $0.9 \% \mathrm{p} / \mathrm{v}$ (solución salina), llamada «normal»o «fisiológica» (Aldrete et al., 2004) se originó durante la pandemia de cólera que devastó Europa en 1831; no obstante, a pesar de su continuo y generalizado uso médico en la reposición y mantenimiento de fluidos humanos, los orígenes de esta solución permanecen oscuros (Awad et al., 2008).

Los animales de agua dulce mantienen sus fluidos internos a niveles hiperosmóticos en relación con el agua dulce (Hill et al., 2006). Por otro lado, la mayoría de los invertebrados marinos son aproximadamente isoosmóticos con el agua de mar, por lo que se les pueden denominar eurihalinos osmoconformadores (Beadle, 1957; Hill,
1979; Barja, 1993; Castelló, 1993; MaedaMartínez, 2002; Hill et al., 2006; Campbell y Reece, 2007; Fanjul y Hiriart, 2008). Los peces marinos teleósteos son reguladores hipoosmóticos, en los cuales la presión osmótica del tejido es mucho más baja que el agua en la que viven (Hill et al., 2006).

Existe una escasez de literatura sobre el mantenimiento de larvas vivas de cestodos de hospedadores marinos. En un trabajo reciente realizado con plerocercoides de bivalvos marinos (Castro et al., 2019), se observó que después de solo cuatro horas de mantenerlos en solución salina fisiológica normal-SSF - $(0.85 \%)$, perdían su vitalidad, se volvían lentos y se hinchaban, lo que impedía lograr una postura óptima para su fijación en glutaraldehído, comprometiendo la calidad de las imágenes SEM del microscopio electrónico de barrido. Para corregir este problema 
Cuadro 1. Parámetros fisicoquímicos de las soluciones experimentales

\begin{tabular}{clcc}
\hline Código & \multicolumn{1}{c}{ Solución } & $\mathrm{pH}$ & $\begin{array}{c}\text { Salinidad } \\
(\%)\end{array}$ \\
\hline $\mathrm{A}$ & $\mathrm{SSF}$ & 7.00 & 0.85 \\
$\mathrm{~B}$ & $1 / 4 \mathrm{AM}+3 / 4 \mathrm{SSF}$ & 7.74 & 1.59 \\
$\mathrm{C}$ & $1 / 2 \mathrm{AM}+1 / 2 \mathrm{SSF}$ & 7.89 & 2.27 \\
$\mathrm{D}$ & $3 / 4 \mathrm{AM}+1 / 4 \mathrm{SSF}$ & 7.90 & 2.94 \\
$\mathrm{E}$ & $\mathrm{AM}$ & 7.856 & 3.59 \\
\hline
\end{tabular}

SSF: Solución salina fisiológica; $1 / 4 \mathrm{AM}+3 / 4$ SSF: $1 / 4$ de agua de mar $+3 / 4$ de solución salina fisiológica; $1 / 2 \mathrm{AM}+1 / 2 \mathrm{SSF}$ : agua de mar + solución salina fisiológica en partes iguales; $3 / 4 \mathrm{AM}+$ $1 / 4 \mathrm{SSF}: 3 / 4$ de agua de mar $+1 / 4$ de solución salina fisiológica; AM: Agua de mar

$\mathrm{y}$ tener larvas con mejor supervivencia y condición física, se han evaluado cinco soluciones salinas a dos temperaturas: ambiental y a temperatura constante $0.5^{\circ} \mathrm{C}$. El objetivo de este trabajo fue elaborar una solución que permita extender la supervivencia de estos plerocercoides para su examen morfológico, sin usar un medio de cultivo, lo que es importante cuando el investigador tiene pocas muestras para llevar a cabo su estudio; además de evaluar la temperatura más favorable.

\section{Materiales y Métodos}

Se usaron cinco soluciones: A, B, C, D y E (Cuadro 1), tanto para el tratamiento 1 (T-1) a temperatura ambiente como para el tratamiento $2(\mathrm{~T}-2)$ a $0.5{ }^{\circ} \mathrm{C}$, lo que hace un total de 10 pruebas en las que se usaron 100 plerocercoides vivos, 10 para cada prueba. Los plerocercoides utilizados fueron identificados como «Tetraphyllidea» de acuerdo con Caira et al. (2017) del género Caulobothrium (Castro et al., 2019) y se extrajeron de la gónada de una concha de abanico Argopecten purpuratus Lamarck, 1819, de la bahía de Sechura, Piura, Perú.
Las soluciones con los diferentes parámetros fisicoquímicos del agua se muestran en el Cuadro 1. El pH de estas soluciones se midió con un multiparámetro Handy Lab680 de SI Analytics, y la salinidad con un medidor de conductividad InoLab WTW. Los experimentos se llevaron a cabo en el Laboratorio de Patobiología Acuática del Instituto del Mar del Perú (IMARPE-Callao).

Las soluciones utilizadas se esterilizaron en autoclave a $121^{\circ} \mathrm{C}$ durante $15 \mathrm{~min}$. El agua de mar empleada para las diferentes soluciones fue filtrada previamente. Las soluciones se colocaron en placas de Petri de $5.4 \mathrm{~cm}$ de diámetro y con 10 plerocercoides en cada una. Las unidades de medida fueron la movilidad, forma y supervivencia de los plerocercoides obtenidas por observación (Figuras 1-2). El movimiento normal del plerocercoide significa estiramiento y contracción del cuerpo y de los botridios, desde una posición de reposo hasta el máximo (Figura $3)$. Cada placa se evaluó a la misma hora en diferentes días (Figuras 1-2). En T-2, las placas se colocaron a temperatura ambiente $\left(27.3{ }^{\circ} \mathrm{C}\right)$ durante 1 hora antes de proceder a la evaluación del estado de los plerocercoides. 

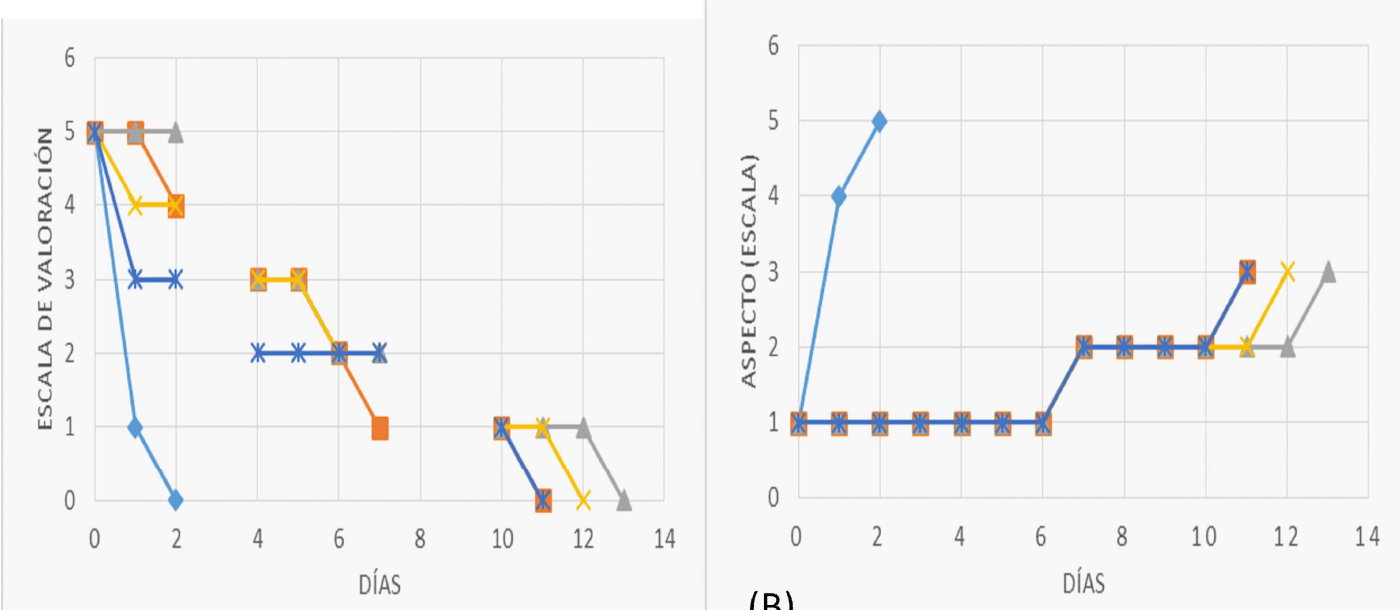

(B)

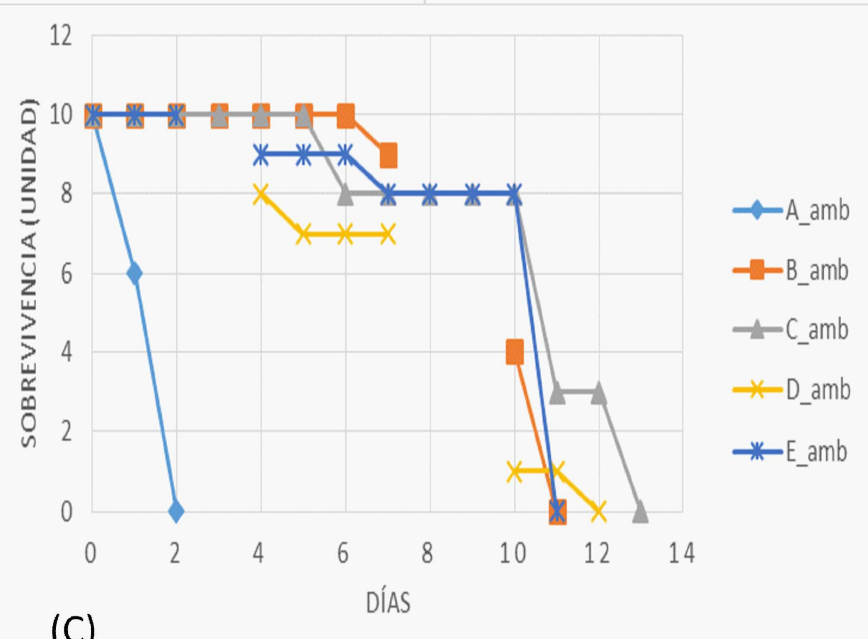

(C)

Figura 1. Tratamiento 1. Cambios en (A) Movimiento, (B) Forma y (C) Sobrevivencia en los plerocercoides en cinco rangos de salinidad a temperatura ambiente (Lectura: $4 \mathrm{pm}$, $27.3^{\circ} \mathrm{C}$ ).

Categoría A: Solución salina fisiológica; B: $1 / 4 \mathrm{AM}+3 / 4 \mathrm{SSF}$ : 1/4 de agua de mar +3/4 de solución salina fisiológica; $\mathrm{C}: 1 / 2 \mathrm{AM}+1 / 2 \mathrm{SSF}$ : agua de mar + solución salina fisiológica en partes iguales; D: $3 / 4 \mathrm{AM}+1 / 4 \mathrm{SSF}$ : $3 / 4$ de agua de mar $+1 / 4$ de solución salina fisiológica; E: Agua de mar

Escala de movimiento: 5: muy activo, 4: activo, 3: moderadamente activo, 2: apenas activo, 1: muy lento.

Escala de forma: 1: $\mathrm{N}$ (normal), 2: LE (ligeramente encogido), 3: $\mathrm{E}$ (encogido), 4: $\mathrm{H}$ (hinchado), 5: HE (hinchado, encogido)

Se utilizó un análisis de Kruskal-Wallis para identificar diferencias entre las soluciones utilizadas. Complementariamente se utilizó el test estadístico Dunn para hacer comparaciones múltiples entre las respuestas evaluadas (movimiento, forma y sobrevivencia) de los tratamientos utilizados.

\section{Resultados}

Las soluciones B_amb y C_amb (T-1) permitieron una mayor supervivencia de los plerocercoides $(p<0.05)$ en comparación con el resto de las soluciones, no habiendo diferencia significativa entre ellos (Cuadro 2). 

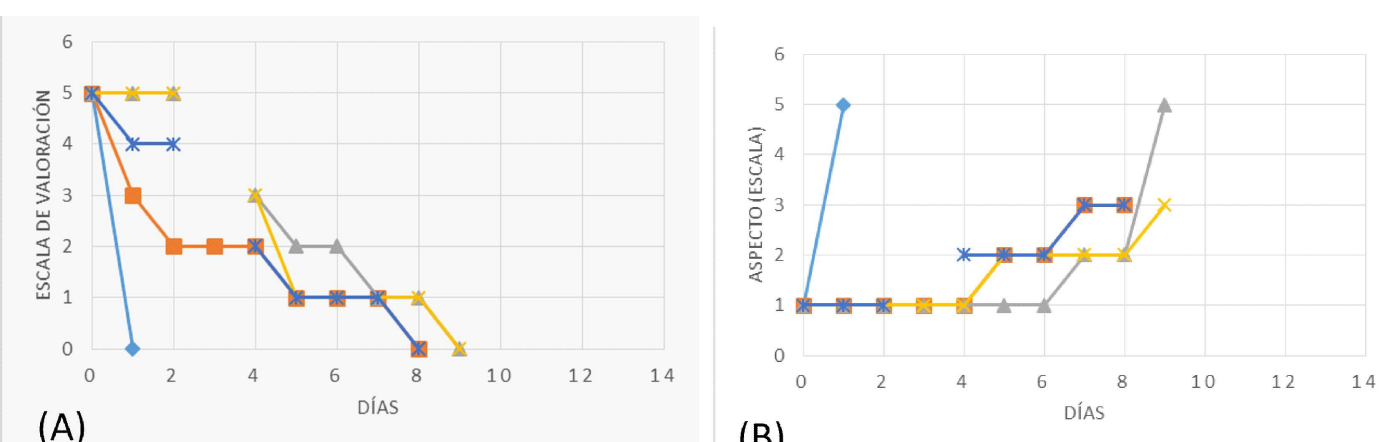

(B)

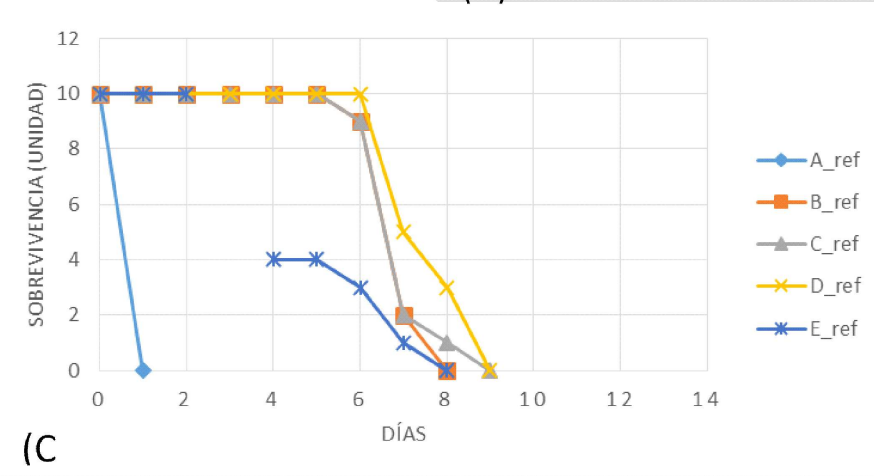

Figura 2. Tratamiento 2. Cambios en (A) Movimiento, (B) Forma y (C) Supervivencia en los plerocercoides en cinco rangos de salinidad a temperatura constante (Lectura: $4 \mathrm{pm}$, $\left.0.5^{\circ} \mathrm{C}\right)$.

Categoría A: Solución salina fisiológica; B: $1 / 4 \mathrm{AM}+3 / 4 \mathrm{SSF}$ : 1/4 de agua de mar +3/4 de solución salina fisiológica; $\mathrm{C}: 1 / 2 \mathrm{AM}+1 / 2 \mathrm{SSF}$ : agua de mar + solución salina fisiológica en partes iguales; D: $3 / 4 \mathrm{AM}+1 / 4 \mathrm{SSF}: 3 / 4$ de agua de mar $+1 / 4$ de solución salina fisiológica; E: Agua de mar

Escala de movimiento: 5: muy activo, 4: activo, 3: moderadamente activo, 2: apenas activo, 1: muy lento.

Escala de forma: 1: $\mathrm{N}$ (normal), 2: LE (ligeramente encogido), 3: E (encogido), 4: H (hinchado), 5: HE (hinchado, encogido)

En T-1 (Figura 1), los plerocercoides estaban vivos en las soluciones B amb, C_amb, D_amb y E_amb en el segundo día, pero solo aquellos que estaban en la solución $\mathrm{C}$ amb se mantuvieron muy activos. En el caso de las soluciones $\mathrm{B}$ amb y $\mathrm{C}$ amb, los plerocercoides estaban vivos al sexto día, sin cambios en su morfología, pero con poca actividad. De otra parte, tres plerocercoides permanecieron vivos hasta el día 12 en la solución C_amb, mostrando cambios en su morfología (ligeramente encogidos) y con una actividad muy lenta.

En la Figura 2, se puede observar que para $\mathrm{T}-2$, los plerocercoides se encontraban vivos en las soluciones B_ref, C_ref, D_ref y E_ref al segundo día, pero solo aquellos que estaban en la solución $\mathrm{C}$ _ref se mantuvieron muy activos. En el cuarto día, los plerocercoides de las soluciones B_ref, C_ref y D_refno presentaron cambios morfológicos 
Cuadro 2. Comparación de dos tratamientos: T-1 a temperatura ambiente y T-2 a temperatura constante $0.5^{\circ} \mathrm{C}$ con cinco soluciones (A, B, C, D y E) con relación a la supervivencia de los plerocercoides

\begin{tabular}{|c|c|c|c|c|c|c|c|c|c|}
\hline & \multicolumn{9}{|c|}{ Medios salinos a temperatura ambiental $\left(27.3^{\circ} \mathrm{C}\right)$ y a $0.5^{\circ} \mathrm{C}$} \\
\hline & A_amb & A_ref & B_amb & B_ref & C_amb & C_ref & D_amb & D_ref & E_amb \\
\hline \multirow[t]{2}{*}{ A_ref } & 0.47 & & & & & & & & \\
\hline & 0.32 & & & & & & & & \\
\hline \multirow[t]{2}{*}{ B_amb } & -4.94 & -5.41 & & & & & & & \\
\hline & 0.00 & 0.00 & & & & & & & \\
\hline \multirow[t]{2}{*}{ B_ref } & -2.74 & -3.20 & 2.21 & & & & & & \\
\hline & 0.00 & 0.00 & 0.01 & & & & & & \\
\hline \multirow[t]{2}{*}{ C_amb } & -5.18 & -5.64 & -0.23 & -2.44 & & & & & \\
\hline & 0.00 & 0.00 & 0.41 & 0.01 & & & & & \\
\hline \multirow[t]{2}{*}{ C_ref } & -2.78 & -3.24 & 2.17 & -0.04 & 2.40 & & & & \\
\hline & 0.00 & 0.00 & 0.02 & 0.48 & 0.01 & & & & \\
\hline \multirow[t]{2}{*}{ D_amb } & -3.80 & -4.26 & 1.15 & -1.06 & 1.38 & -1.02 & & & \\
\hline & 0.00 & 0.00 & 0.13 & 0.14 & 0.08 & 0.15 & & & \\
\hline \multirow[t]{2}{*}{ D_ref } & -3.29 & -3.76 & 1.65 & -0.56 & 1.88 & -0.52 & 0.50 & & \\
\hline & 0.00 & 0.00 & 0.05 & 0.29 & 0.03 & 0.30 & 0.31 & & \\
\hline \multirow[t]{2}{*}{ E_amb } & -5.00 & -5.46 & -0.05 & -2.26 & 0.18 & -2.22 & -1.20 & -1.70 & \\
\hline & 0.00 & 0.00 & 0.47 & 0.01 & 0.43 & 0.01 & 0.12 & 0.04 & \\
\hline \multirow[t]{2}{*}{ E_ref } & -1.59 & -2.06 & 3.35 & 1.14 & 3.58 & 1.18 & 2.20 & 1.70 & 3.40 \\
\hline & 0.06 & 0.02 & 0.00 & 0.13 & 0.00 & 0.12 & 0.01 & 0.04 & 0.00 \\
\hline
\end{tabular}

Temperatura ambiente: soluciones A_amb, B_amb, C_amb, D_amb y E_amb Temperatura constante $\left(0.5^{\circ} \mathrm{C}\right)$ : soluciones: A_ref, B_ref, C_ref, D_ref, E_ref Categoría A: Solución salina fisiológica; B: 1/4 AM + 3/4 SSF: 1/4 de agua de mar + 3/4 de solución salina fisiológica; C: $1 / 2 \mathrm{AM}+1 / 2 \mathrm{SSF}$ : agua de mar + solución salina fisiológica en partes iguales; D: $3 / 4$ AM + 1/4 SSF: $3 / 4$ de agua de mar + 1/4 de solución salina fisiológica; E: Agua de mar

anormales, pero disminuyeron su actividad. En el quinto día todas las larvas en la solución C_ref estaban viva, sin cambios morfológicos, pero con poca actividad, en tanto que en las soluciones B_ref y D_ref se observó un ligero encogimiento del cuerpo. Solo en la solución D_ref se mantuvieron vivas todas las larvas al sexto día, aunque levemente encogidas y con una actividad muy lenta. Finalmente, solo 1 (C_ref) y 3 (D_ref) plerocercoides permanecieron con vida hasta el octavo día, presentando contracción del cuerpo y lenta actividad.

En la solución A $(0.85 \%$ de solución salina) de T-1, los plerocercoides sobrevivieron solo un día y se hincharon. Esto mismo sucedió en esta solución en T-2, donde tuvieron una supervivencia de solo horas (menos de un día), muriendo hinchados y encogidos. 

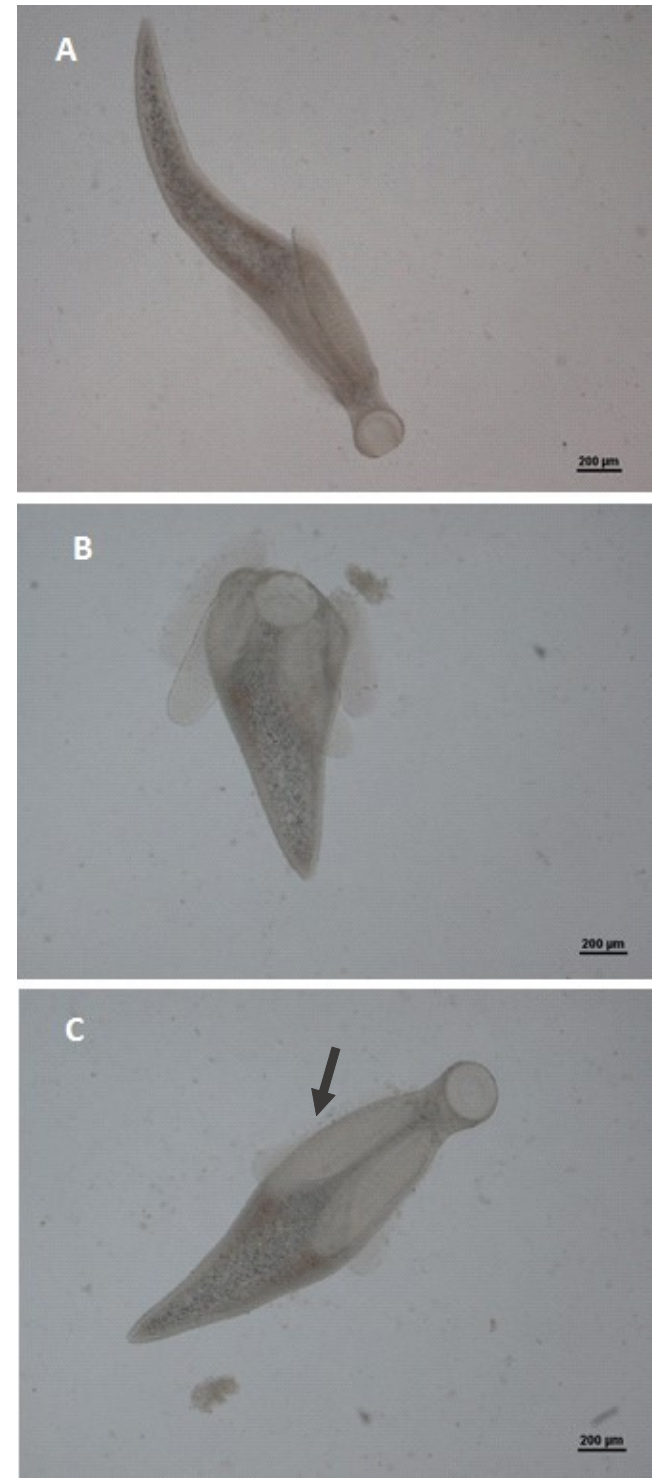

Figura 3. Larva plerocercoide (aumento 4x, barra $200 \mu \mathrm{m}$ ). A. Larva estirada. B. Larva contraída. C. Larva normal en reposo. La flecha muestra un botridio

\section{Discusión}

Aunque la solución salina normal al $0.85 \%$ se usa para el estudio de órganos internos y para el mantenimiento de parásitos en el análisis rutinario de organismos acuáticos (Cabrera et al., 2010; Cribb y Bray, 2010), es claramente inadecuada para el estudio de los plerocercoides encontrados en el bivalvo marino A. purpuratus. En este contexto, Humason (1972) refiere que existen diferentes tipos de soluciones fisiológicas según sea el tipo de investigación y que estas deben ser isotónicas para mantener los tejidos en condiciones normales (las células se hinchan en soluciones hipotónicas y se encogen en las hipertónicas), sugiriendo que la SSF sea de $0.9 \%$ para los mamíferos y de $0.75 \%$ para los invertebrados. Asimismo, Hoffman (1999) menciona que la SSF de $0.8-0.9 \%$ es adecuada para el uso temporal en truchas, ya que la sangre es casi isotónica.

Existe una amplia variedad de soluciones salinas que se utilizan para el estudio parasitológico de organismos acuáticos, como la SSF comúnmente utilizada para helmintos parásitos, que es $0.75 \%$ (Meyer y Penner, 1962; Mendoza-Palmero et al., 2007). Otros, como Eiras et al. (2000) recomiendan una solución fisiológica con un rango de $\mathrm{NaCl}$ de 0.6-0.8\% para la inspección de órganos internos. Según Hoffman (1999), la mayoría de los parásitos internos deben estudiarse con una SSF cuyo rango de cloruro de sodio sea entre $0.8-0.9 \%$, en tanto que Justine et al. (2012) recomiendan el uso de una solución salina al $0.9 \%$ para los parásitos intestinales de los peces. En este sentido, autores como Salgado (2009), Quiroz (2014), Lemus et al. (2016), y Scholz y Kuchta (2005), RodríguezGonzáles y Vidal-Martínez (2008) recomiendan o utilizaron una solución salina de $0.7 \%$ para el examen de los parásitos intestinales y órganos internos.

Asimismo, Morey (2019) estudió los órganos internos de peces amazónicos utilizando agua destilada y Moreno et al. (2008) colocaron parásitos de vísceras en SSF al $0.75 \%$ de peces Mugil liza, Oreochromis mossambicus y Dormitator maculatus; Barrios-Gutiérrez et al, (2018) y Vázquez et al. (2004) analizaron peces dulceacuícolas colocando los órganos internos en SSF al $0.65 \%$; en tanto que una SSF del $0.6 \%$ fue utilizada por Sierra y Lamothe (1990) para el 
estudio de órganos internos del pez marino Pomacanthus arcuatus. Por otro lado, Tharme et al. (1996) utilizaron agua de mar para el estudio del bivalvo Donax serra parasitado con larvas de digeneos; y Morales y Cuéllar (2008) utilizaron agua de mar estéril para el estudio de los órganos internos del camarón peneido en general.

Cabe señalar que no se menciona el tipo de solución salina utilizada para la recolección de parásitos en la mayoría de los artículos de investigación, posiblemente debido a que son fijados de manera inmediata, sin hacer un minucioso estudio in vivo previo. Aunque la mayoría de los investigadores consideran la SSF como una solución estándar para el estudio de los parásitos de helmintos internos de cualquier organismo acuático (peces, moluscos o crustáceos) de agua dulce, los hallazgos en este trabajo demuestran que otras formulaciones básicas pueden dar mejores resultados.

Claramente, la solución salina estándar, representada en este estudio por la solución «A», $(0.85 \% \mathrm{NaCl})$ no es recomendable ya que los plerocercoides no sobreviven más de un día y sus cuerpos se deforman debido a la baja cantidad de sal en la solución en las dos temperaturas evaluadas. En el presente estudio no se tomó en cuenta la turbidez de las soluciones con el pasar de los días; sin embargo se debe tener en cuenta este factor, ya que el agua se enturbia al permanecer los plerocercoides muertos en el agua, lo que influye negativamente en la supervivencia de las demás larvas. Por otro lado, mantener las soluciones transparentes con el cambio de una nueva solución por día, mejora la supervivencia y la vitalidad de las larvas plerocercoides de concha de abanico.

Anantaraman (1963), en un trabajo similar realizado con larvas de plerocercoides de peces, mostró que por debajo del $50 \%$ de agua de $\operatorname{mar}(25 \%, 10 \%$ y agua corriente), el agua pasa a los tejidos cuando se reduce la presión osmótica del medio externo produ- ciendo la hinchazón y la posterior muerte de la larva. En este trabajo se pudo observar que la solución A (SSF) tuvo una reacción similar con soluciones por debajo del $50 \%$.

Se puede concluir que las soluciones B y C del T-1 son adecuadas para la sobrevivencia de plerocercoides en bivalvos marinos; sin embargo, en la solución C $(2.27 \%$ de salinidad), para las dos temperaturas en evaluación, se pudo observar un mejor tiempo de supervivencia y de vitalidad en los plerocercoides. Esto se aproxima al valor sugerido por Hoar y Hickman (1975), quienes encontraron que lo más apropiado para crustáceos marinos era una solución de $\mathrm{NaCl}$ al $2.67 \%$. Posiblemente, la solución «C» es isotónica con el plerocercoide, pero se necesitaría de estudios adicionales para demostrarlo.

\section{Agradecimientos}

Agradecemos a Arturo Aguirre-Velarde por el aporte estadístico y revisión del documento. También agradecemos a Steve Webb, Cawthron Institute, Nelson, Nueva Zelanda, por su asesoramiento.

\section{Literatura Citada}

1. Aldrete A, Guevara U, Capmourteres EM. 2004. Texto de anestesiología teórico-práctica. $2^{\mathrm{a}}$ ed. México DF: Manual Moderno. 1668 p.

2. Anantaraman S. 1963. Volume regulation in tetraphyllidean larvae. Proc Indian Academy Sci - Section B 57: 117123. doi: 10.1007/BF03052011

3. Awad S, Allison SP, Lobo DN. 2008. The history of $0.9 \%$ saline. Clin Nutr 27: 179-188. doi: 10.1016/j.clnu.2008.01.008

4. Barja G 1993. Fisiología animal y evolución. Fuenlabrada, Madrid: EdAkal. 137 pp.

5. Barrios-Gutiérrez JJ, MartínezRamírez E, Gómez-Ugalde RM, García-Varela M, Pinacho-Pinacho CD. 2018. Helmintos parásitos de los 
peces dulceacuícolas de la Reserva de la Biosfera Tehuacán-Cuicatlán, región Oaxaca. Rev Mex Biodiv 89: 29-38.

6. Beadle LC. 1957. Comparative physiology: osmotic and ionic regulation in aquatic animals. Annu Rev Physiol 19: 329-58. doi: 10.1146/annurev.ph.19.030157.001553

7. Cabrera L, Diaz S, Parra K et al. 2010. Detección de parásitos protozoarios y helmintos en el molusco bivalvo Geukensia demissa (Dillwyn, 1817) presente en el sector de Nararét del Municipio Mara, Estado Zulia, Venezuela. Rev Cient (Maracaibo) 20: 7-16.

8. Caira JN, Jensen K, Ruhnke TR. 2017. «Tetraphyllidea» van Beneden, 1850 relics. In: Caira JN, Jensen K (eds). Planetary biodiversity inventory (20082017): tapeworms from vertebrate bowels of the earth. The University of Kansas, Natural History Museum, Special Publication N. ${ }^{\circ} 25$ p 371-400.

9. Campbell N, Reece J. 2007. Biología. Madrid, España: Ed Médica Panamericana. $1532 \mathrm{p}$.

10. Castelló F. 1993. Acuicultura marina: fundamentos biológicos y tecnología de la producción. España: Universitat de Barcelona. $739 \mathrm{p}$.

11. Castro T, Mateo DR, Greenwood SJ, Mateo EC. 2019. First report of the metacestode Caulobothrium sp. in the Peruvian scallop Argopecten purpuratus from Sechura Bay, Piura, Peru. Parasitol Res 118: 2369-2376 doi: 10.1007/s00436-019-06361-3

12. Cribb TH, Bray RA. 2010. Gut wash, body soak, blender, and heat-fixation: approaches to the effective collection, fixation and preservation of trematodes of fishes. Syst Parasitol 76: 1-7. doi: 10.1007/s11230-010-9229-z

13. Eiras JC, Takemoto RM, Pavanelli GC. 2000. Métodos de estudio y técnicas laboratoriales en parasitología de peces. Zaragoza, España: Acribia. 133 p.

14. Fanjul ML, Hiriart M. 2008. Biología funcional de los animales. $2^{\circ}$ ed. México: Siglo XXI. 399 p.
15. Hill RW, Wyse GA, Anderson M. 2006. Fisiología animal. Madrid, España: Ed Médica Panamericana. $1038 \mathrm{p}$.

16. Hill $\boldsymbol{R W}$. 1979. Fisiología animal comparada: un enfoque ambiental. España: Ed Reverté. 910 p.

17. Hoar WS, Hickman CP. 1975. A laboratory companion for general and comparative physiology. Prentice-Hall. $297 \mathrm{p}$.

18. Hoffman GL. 1999. Parasites of North American freshwater fishes. USA: Cornell University Press. 539 p.

19. Humason GL. 1972. Animal tissue techniques. $3^{\text {rd }}$ ed. San Francisco, USA: WH Freeman. $641 \mathrm{p}$.

20. Justine JL, Briand MJ, Bray RA. 2012. A quick and simple method, usable in the field, for collecting parasites in suitable condition for both morphological and molecular studies. Parasitol Res 111: 341-351. doi: 10.1007/s00436-012-2845-6

21. Lemus EG, Mejia AG, Rodriguez MG 2016. Prevalencia de parásitos helmintos en peces de agua dulce del embalse Cerrón Grande de El Salvador. Tesis de Médico Veterinario Zootecnista. El Salvador: Univ. de El Salvador. 55 p.

22. Maeda-Martinez AN. 2002. Los moluscos pectínidos de Iberoamérica: ciencia y acuicultura. México: Ed Limusa. $501 \mathrm{p}$.

23. Mendoza-Palmero CA, EspinosaPérez H, Salgado-Maldonado G. 2007. Helmintos parásitos de peces dulceacuícolas. En: A. Lot (ed). Guía Ilustrada de la Cantera Oriente: caracterización ambiental e inventario biológico. México: Univ. Nacional Autónoma de México. p 179-191.

24. Meyer MC, Penner LR. 1962. Laboratory essentials of parasitology. USA: WMC Brown. 134 p.

25. Morales V, Cuéllar-Anjel J. 2008. Guía técnica - Patología e inmunología de camarones penaeidos. Panamá, Rep. de Panamá: Programa CYTED Red II-D Vannamei. $270 \mathrm{p}$. 
26. Moreno AZ, Fuentes JL, Mago Y, Chinchilla O. 2008. Descripción, taxonomía e índices ecológicos de parásitos en peces de la laguna de los Mártires, Isla de Margarita, Venezuela. Saber. Universidad de Oriente Venezuela 20(1): 3-11.

27. Morey GAM. 2019. Parasitología en peces de la Amazonía. Fundamentos y técnicas parasitológicas, profilaxis, diagnóstico y tratamiento. Iquitos, Perú: Instituto de Investigaciones de la Amazonía Peruana (IIAP). $100 \mathrm{p}$.

28. Quiroz LK. 2014. Estudio de la parasitofauna en el jurel (Trachurus picturatus murphyi, Chirichigno y Vélez 1998), con énfasis en zoonosis parasitaria. Tesis de Ingeniero Pesquero. Lima, Perú: Univ. Nacional Agraria La Molina. 81 p.

29. Rodríguez-González, A, Vidal-Martínez VM. 2008. Las comunidades de helmintos del lenguado (Symphurus plagiusa) en la costa de Campeche, México. Rev Mex Biodiv 79: 159-173.

30. Salgado G. 2009. Manual de prácticas de parasitología con énfasis en helmintos parásitos de peces de agua dulce y otros ani- males silvestres de México. México: Univ. Nacional Autónoma de México 56 p.

31. Scholz T, Kuchta R. 2005. Parásitos metazoarios de peces nativos y de cultivo en amazonia, Perú. Iquitos, Perú: Academia de Ciencias de la Republica Checa, Gobierno Regional de la Región de Loreto, Universidad Nacional de la Amazonía Peruana.105 p.

32. Sierra N, Lamothe R. 1990. Identificación taxonómica del trematodo Cricocephalus albus en el pez Pomacanthus arcuatus capturado en el Caribe Mexicano. Téc Pec Méx 28: 165-172.

33. Tharme RE, Webb SC, Brown AC. 1996. Organisms associated with the sandy-beach bivalve Donax serra Röding, with a description of Cercaria serrae sp. nov. (Trematoda). S Afr J Zool 31: 86-90. doi: 10.1080/02541858.1996.11448398

34. Vázquez R, Ramírez J, Osorio D, Chávez LA, Constantino F. 2004. Lesiones causadas por helmintos del aparato digestivo en peces estuarinos de la Laguna Tres Palos, Guerrero, México. Vet Méx 35: 369-378. 